\title{
Hospital-level balloon tamponade use is associated with increased mortality for all patients presenting with acute variceal haemorrhage
}

\author{
Elliot B. Tapper ${ }^{1,2,3}$ (D) | Ghideon Ezaz ${ }^{4}$ | Vilas Patwardhan ${ }^{4}$ | Jessica Mellinger ${ }^{1,2}$ | \\ Alan Bonder $^{4}$ | Michael Curry ${ }^{4}$ Sameer D. Saini ${ }^{1,2,3,5}$
}

${ }^{1}$ Division of Gastroenterology and Hepatology, University of Michigan, Ann Arbor, MI, USA

${ }^{2}$ Institute for Healthcare Policy and Innovation, Ann Arbor, MI, USA

${ }^{3}$ Veterans Affairs Hospital, Ann Arbor, MI, USA

${ }^{4}$ Division of Gastroenterology and Hepatology, Beth Israel Deaconess Medical Center, Harvard Medical School, Boston, MA, USA

${ }^{5}$ Veterans Affairs Center for Clinical Management Research (CCMR), Ann Arbor, MI, USA

\section{Correspondence}

Elliot B. Tapper, Taubman, Ann Arbor, MI, USA. Email: etapper@umich.edu

Handling Editor: Christophe Bureau

\begin{abstract}
Background \& Aims: Balloon tamponade (BT) can bridge patients to salvage therapy for uncontrollable acute variceal haemorrhage (AVH). However, data are limited regarding the reasons for, rate of and outcomes associated with Balloon tamponade use. Methods: First, we performed an single-centre cohort study of all patients $(N=139)$ with oesophageal acute variceal haemorrhage from $01 / 2009$ to $10 / 2015$. Associations between Balloon tamponade use and adherence to four quality metrics (endoscopy within 12 hours, band-ligation, pre-endoscopy antibiotics and octreotide) were evaluated. Second, we analysed the National Inpatient Sample (2005-2011) to determine the association between in-hospital mortality for patients and their hospital's Balloon tamponade-utilization to acute variceal haemorrhage volume ratio.

Results: In the national cohort, 5.5\% of 140521 acute variceal haemorrhage admissions required Balloon tamponade utilization. Adjusting for patient- and hospital-level confounders, the rate of Balloon tamponade use per acute variceal haemorrhage managed at any given hospital was associated with increased mortality for all-comers with acute variceal haemorrhage. Compared to the lowest tertile, acute variceal haemorrhage admissions in the highest Balloon tamponade utilizers were associated with increased mortality of (OR1.17 95\% Cl (1.01-1.37).

In the single-centre cohort, 14 (10.1\%) patients required Balloon tamponade. Balloon tamponade utilization was significantly associated with alcohol abuse $(50.4 \%$ vs $21.4 \%$, $P=.04)$, hepatocellular carcinoma (35.7\% vs 8.8\%, $P=.01$ ), higher median model for end-stage liver disease (MELD) score (26.3vs15.5, $P=.002)$ and active bleeding during endoscopy (64.3\% vs $27.5 \%, P=.01)$. Failure to provide all quality metrics was associated with a higher model for end-stage liver disease-adjusted risk of Balloon tamponade use: OR $16.795 \% \mathrm{Cl}(4.17-100.0, P<.0001)$.

Conclusion: Balloon tamponade use is associated with severity of bleeding but may also implicate deficits in processes of care. Even for patients who did not need Balloon tamponade, presentation to hospitals with high Balloon tamponade utilization increases their odds of dying from acute variceal haemorrhage.

KEYWORDS

cirrhosis, endoscopy, liver disease, model for end-stage liver disease score, portal hypertension
\end{abstract}




\section{1 | INTRODUCTION}

Over the last 40 years, the prognosis for patients with acute variceal haemorrhage $(\mathrm{AVH})$ has improved dramatically. ${ }^{1-3}$ Contemporary outcomes, including $17.0 \%$ 6-week mortality, ${ }^{4}$ compare favourably with the $>40 \%$ mortality observed as late as the early 1980 s when balloon tamponade (BT) was often a primary therapy. ${ }^{2}$ These improvements can be attributed in large part to technical innovations including timely endoscopy with band ligation, vasoactive medications and prophylactic antibiotics. ${ }^{5}$ Given the availability of these therapies which now constitute the standard of care, BT is currently reserved for patients too unstable for endoscopy or for whom endoscopic therapy has failed or is technically impossible. In this context, BT utilization is an important sentinel event, one that reflects both a high risk of mortality for the patient and indicates, in some cases, suboptimal AVH management. Data regarding the contemporary rate and reasons for BT utilization, however, are limited.

Some proportion of BT utilization may be preventable, particularly if the standard of care is not provided. For example a patient with AVH who fails to receive timely endoscopy or a somatostatin analogue may be more likely to develop unstable bleeding requiring BT. So too would the patient of an endoscopist uncomfortable with band-ligation. In these cases, BT use speaks to problems in the delivery of $\mathrm{AVH}$ care. We recently showed that $14 \%$ of all patients with $\mathrm{AVH}$ receive no endoscopic therapy, $9 \%$ do not receive vasoactive medications and $37 \%$ do not receive prophlylactic antibiotics. ${ }^{4}$ We hypothesized that if a patient received BT, many others would have been exposed to similar processes of care and thus higher BT utilization at the hospitallevel would be associated with poor outcomes. Herein we test this hypothesis in a study with two aims: one, to evaluate the rate, clinical associations and impact of BT utilization at the hospital-level and, two, to evaluate in a single-centre study whether BT use reflects gaps in quality of care.

\section{2 | METHODS}

Given our aims, we developed a nationwide cohort and a single-centre cohort of patients with AVH. The National Inpatient Sample (NIS) is a nationally representative administrative database of hospitalizations that offers details of patient demographics and comorbidities as well as procedure utilization (as identified through billing records). These data are useful in determining the clinical associations with BT and the impact of BT utilization on in-hospital mortality. However, the NIS lacks the necessary data to link BT use to established quality metrics in the management of $\mathrm{AVH}$. For this reason, we also examined a single-centre cohort where adherence to quality metrics could be documented and tested for an association with BT utilization.

\section{1 | National cohort}

Second, we used the National Inpatient Sample (years 2005 to 2011) to determine the in-hospital mortality associated with any given

\section{Key points}

- The management of variceal haemorrhage is of variable quality

- Optimal management includes timely endoscopy with band ligation and vasoactive medications

- Balloon tamponade (BT) is reserved for bleeding refractory to endoscopic and medical management

- Balloon tamponade may be associated with gaps in the quality of bleeding management

- The rate of BT varies substantially across high volume hospital

- Mortality after variceal bleeding without BT use is higher at hospitals with higher overall BT use

hospital's ratio of BT utilization (ICD-9 CM 96.06) to the volume of patients with a primary diagnosis of AVH (ICD-9 456.0 or 456.20) or a secondary diagnosis of AVH with a primary diagnosis of cirrhosis (ICD-9 $571.2571 .5,571.6$ ) or its complications including portal hypertension (ICD-9 572.3), hepatic encephalopathy (HE; ICD-9 572.2), ascites (ICD-9 789.59) or hepatorenal syndrome (ICD-9 572.4). The analysis was restricted to hospitals that care for more than 10 patients with $\mathrm{AVH}$ per year.

We first compared patient- and hospital-level factors associated with BT use. Patient-level included demographics (gender, race, age, socioeconomic status), insurance payor, aetiology of liver disease (alcohol, viral), liver specific comorbidities (ascites, HE, hepatocellular carcinoma-HCC) and Charlson score. ${ }^{6}$ Hospital-level factors included size, transfer-rate and teaching status. We also documented receipt of concurrent treatments to manage variceal haemorrhage including upper endoscopy (ICD-9 45.13, 44.13, 42.23, 42.33) and transjugular intrahepatic portosystemic shunt (TIPS) (ICD-9 39.1).

Next, we classified hospitals according to rate of BT use. Hospitallevel factors were evaluated on an annual basis given that the NIS only allows for hospital level analysis for each year. BT utilization was divided into four groups: those with zero BT utilization in a given year and three other groups classified according tertiles of BT use per AVH admission.

Our primary outcome was in-hospital mortality. We estimated the odds ratio (OR) of mortality associated with being admitted to a hospital in the highest tertile of BT utilization and being admitted to a hospital in the lowest tertile of BT utilization. We also compared the effect of being admitted to highest tertile of BT-users to hospitals without documented BT use. These analyses were adjusted for confounders as described below.

\section{2 | Single-centre cohort}

We also analysed a single-centre cohort drawn from Beth Israel Deaconess Medical Center, an academic tertiary care and liver transplant centre, to describe the patient and physician-level factors 
associated with the outcomes of oesophageal AVH from January 2009 through October 2015. The data were collected prospectively from February 2015 forward and retrospectively otherwise. The Institutional Review Board of Beth Israel Deaconess Medical Center approval with a waiver of informed consent. Patients were identified by electronically searching administrative data for inpatient endoscopies performed for bleeding varices (all inpatient procedures performed for ICD-9 456.0 and/or by the known roster of hepatologists over the study period), as well as consult service rolls. Patients transferred following stabilization or management at referring institutions were excluded. Patients with gastric variceal haemorrhage were excluded to simplify the interpretation of the data. Demographic and clinical details were collected as detailed in Table 1.

In this centre, all gastrointestinal haemorrhage was managed by gastroenterology fellows in concert with specialized hepatologists if cirrhosis was established or suspected and general academic gastroenterologists otherwise-all of whom were familiar with $\mathrm{AVH}$ treatment protocols. Patients with $\mathrm{AVH}$ were triaged to the intensive care unit unless haemodynamically stable without transfusion needs. Endotracheal intubation was performed in the setting of active haematemesis and, if not already present, before the placement of a Sengstaken-Blakemore tube for BT. BT insertion was guided by a standardized protocol and documented using template standardized notes. Protocol specified that the gastric balloon alone be inflated to 300-400 cc without use of the oesophageal balloon unless fresh blood is aspirated while the balloon is under $0.5 \mathrm{~kg}$ of tension, and that the gastric balloon be deflated in a stepwise fashion after at least 12 hours of tamponade. Subsequent management (ie repeat endoscopy, TIPS or comfort care) was determined by the hepatology team in concert with the patient's family.

The primary outcome was BT utilization. Secondary outcomes included 6-week mortality and overall transplant-free survival. Survival was confirmed by medical record review and a validated search of the Social Security Death Index. ${ }^{7}$ Transplantation was confirmed by medical record review and a search of an integrated pharmacy database. Exposure variables included patient factors (demographics, preprocedure model for end-stage liver disease (MELD), cirrhotic decompensation and liver cancer) and physician factors (adherence to quality metrics including band ligation, timely endoscopy ( $<12$ hours) and initiation of octreotide and antibiotics prior to endoscopy). The timing of treatments provided was confirmed by reviewing pharmacy dispensing records and nursing notations. The performance of band-ligation was defined as the placement of a band with cessation of haemorrhage at the time of the procedure.

\section{3 | Data analysis}

\subsection{1 | Single-centre cohort}

Statistical analyses included Fisher's exact test, Student's $T$ test and Wilcoxon Rank-Sums test for pairwise comparisons of categorical variables, parametric, and non-parametric continuous variables respectively. A stepwise backward logistic regression procedure was performed to determine the adjusted effect on BT utilization on any variable found to be significant (two-tailed $P<.05$ ) in the pairwise comparisons (see Table 1). Since BT use was rare, analyses were performed using the Firth method for bias reduction. ${ }^{8}$ Given observed numerical (but not statistical) differences in three of the quality metrics between $B T$ recipients and non-recipients, we created a combined binary ( 1 vs 0 ) exposure variable reflecting whether a patient received all four quality metrics and then repeated the regression procedure. All single centre analyses were performed using JMP Pro 12 statistical software.

\subsection{2 | Nationwide cohort}

Two-tailed tests of significance in the comparison of means and proportions were performed using t-tests taking into account survey design (significance threshold $P<.05$ ). Multivariable logistic regression was performed to adjust for the impact of BT use:AVH ratio on in-hospital mortality by taking into account patient factors (age, sex, income, race, insurer, Charlson comorbidity index and liver disease characteristics including the presence of alcoholic cirrhosis, HE, ascites and HCC), management factors (receipt of endoscopy or TIPS) and hospital characteristics (bed size, teaching status,). Analyses were performed using Stata.

\section{3 | RESULTS}

\subsection{National cohort}

Overall, we included 140521 admissions for AVH in our study. Table 1 delineates the differences between patients who did and did not receive BT during their hospitalization for $\mathrm{AVH}$. In general, patients receiving BT were more to be younger, African American and have a history of alcoholic cirrhosis and HE. Notably, they were not less likely to have received an endoscopy.

Table 2 divides the patients into tertiles of their hospital's ratio of BT utilization to AVH volume. Most patients were cared for in hospitals without reported BT use. Among hospitals with BT utilization, there were significant differences in teaching status, hospital size, payer mix and discharge disposition. There was no difference in endoscopy or TIPS utilization. There were increasing unadjusted mortality rates across tertiles that were not statistically significant.

The risk of in-hospital mortality associated with hospital-level BT utilization was examined in Table 3. As expected, cirrhotic comorbidities were associated with increased risk of mortality as was TIPS use while endoscopy was inversely associated. After adjusting for patient-level factors, management details and hospital characteristics, compared to hospitals with low BT use, high BT utilization hospitals were associated with an odds ratio of $1.1795 \% \mathrm{Cl}(1.01-1.37)$ for inhospital mortality each patient with AVH. In a sensitivity analysis, we compared the adjusted mortality rate associated with being admitted to hospitals with highest tertile of BT utilization compared to those without BT use (odds ratio $1.2095 \% \mathrm{Cl}$ (1.10-1.32). Adjusting for year of service did not alter the results. 
TABLE 1 Population characteristics

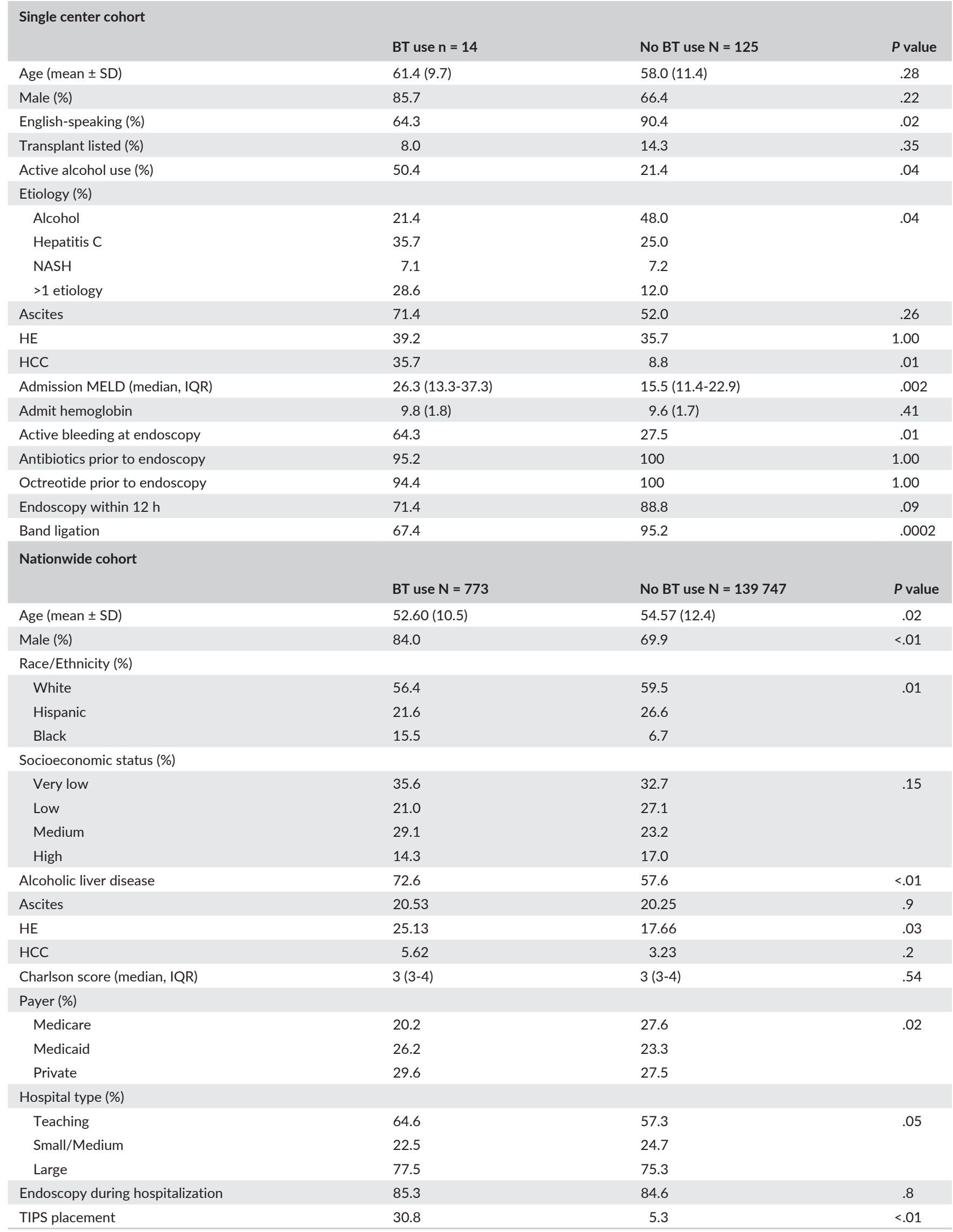

BT, balloon tamponade; HE, Hepatic encephalopathy; HCC, Hepatocellular carcinoma; IQR, interquartile range; MELD, model for endstage liver disease; $\mathrm{NASH}$, nonalcoholic steatohepatitis; TIPS, transjugular intrahepatic portosystemic shunt. 
TABLE 2 Nationwide cohort characteristics by hospital-level bt utilization

\begin{tabular}{|c|c|c|c|c|c|}
\hline In hospital death & 8.1 & 10.9 & 11.8 & 14.0 & .12 \\
\hline SNF & 7.1 & 6.9 & 6.6 & 8.1 & \\
\hline Transfer & 3.4 & 1.7 & 3.8 & 5.8 & \\
\hline Other & 2.6 & 2.2 & 2.2 & 2.3 & \\
\hline \multicolumn{6}{|l|}{ Payer } \\
\hline Self-pay & 13.1 & 12.1 & 12.0 & 17.1 & \\
\hline Other & 8.5 & 10.6 & 7.7 & 5.4 & \\
\hline \multicolumn{6}{|l|}{ Hospital type } \\
\hline Teaching & 55.2 & 79.6 & 73.4 & 52.1 & .001 \\
\hline Small/Medium & 25.8 & 10.3 & 17.2 & 34.5 & $<.0001$ \\
\hline Large & 74.2 & 89.7 & 82.8 & 65.5 & \\
\hline \multicolumn{6}{|l|}{ Interventions } \\
\hline Endoscopy & 84.6 & 84.5 & 85.4 & 86.0 & .56 \\
\hline
\end{tabular}

BT, balloon tamponade; SNF, skilled nursing facility; TIPS, transjugular intrahepatic portosystemic shunt.

\section{2 | Single-centre cohort}

We analysed a single-centre cohort to assess the reasons for BT utilization. Fourteen (10.1\%) patients with oesophageal AVH required BT. (Table 1) Patients requiring BT were more likely to be non-English speaking, actively drinking alcohol, have comorbid liver cancer, present with a substantially higher MELD score and were more likely to be actively bleeding at the time of endoscopy. Patients receiving BT were more likely to experience 6-week mortality, with substantially reduced overall transplant-free survival. While patients receiving BT were numerically but not significantly less likely to receive timely endoscopy, octreotide and antibiotics, they were significantly less likely to have received band ligation. In a logistic regression, MELD and band-ligation were significantly associated with BT utilization. Adjusting to MELD, failure to band was associated with an odds ratio of $8.9495 \% \mathrm{Cl}(1.87-44.4)$ for BT utilization $(P=.007)$. When complete adherence to each of the quality measures was provided, it was associated with a lower MELD-adjusted risk of BT use: $0.0695 \% \mathrm{Cl}$ (0.01-0.24), $P<.0001$.

We investigated the circumstances and outcomes of BT use. Of the 14 patients needing BT, the endoscopic therapy provided was as follows: eight failed attempts at banding on non-bleeding varices at the time of endoscopy (slipped band placement with recurrent haemorrhage), five no treatment, one sodium morrhuate injection and one cyanoacrylate glue injection. The patients receiving not endoscopic therapy as well as those receiving sclerotherapy and glue were actively bleeding with a reported failure to visualize a culprit vessel. Seven (50\%) had a second endoscopy, including the five patients with $\mathrm{HCC}$, all of whom died during their hospitalization. Of the remaining seven, each received a TIPS and six survived to discharge. One patient experienced a complication (oesophageal perforation) owing to BT that was managed conservatively; this patient is alive after 608 days of follow-up.

\section{4 | DISCUSSION}

Balloon tamponade is a temporizing procedure used to "bridge" patients from uncontrolled variceal haemorrhage to definitive therapy, either a second endoscopy or TIPS. Given the limited contemporary data available on BT use, the authors of the Baveno VI consensus have recommended additional research on this topic. ${ }^{5}$ In this study of nationally representative cohort complemented by granular data from a single tertiary care centre, we show that while BT utilization reflects both severity of liver disease and bleeding, BT use itself may identify opportunities to improve hospital-level 
TAB LE 3 Risk factors for in-hospital mortality in the national cohort

\begin{tabular}{|c|c|c|c|}
\hline \multicolumn{4}{|c|}{ Adjusted risk factors for in-hospital mortality } \\
\hline Odds ratio & \multicolumn{3}{|c|}{$95 \%$ Confidence interval } \\
\hline Alcoholic liver disease & 1.36 & 1.21 & 1.52 \\
\hline Ascites & 1.24 & 1.10 & 1.39 \\
\hline Hepatic encephalopathy & 2.91 & 2.61 & 3.25 \\
\hline Hepatocellular carcinoma & 1.91 & 1.50 & 2.44 \\
\hline \multicolumn{4}{|l|}{ Utilization of bleeding interventions } \\
\hline Endoscopy & 0.47 & 0.42 & 0.53 \\
\hline $\begin{array}{l}\text { Transjugular intrahepatic } \\
\text { portosystemic shunt placement }\end{array}$ & 1.76 & 1.46 & 2.12 \\
\hline $\begin{array}{l}\text { Admitting hospital BT/AVH } \\
\text { Ratio-Third tertile to first } \\
\text { tertile }\end{array}$ & 1.17 & 1.01 & 1.36 \\
\hline
\end{tabular}

$\mathrm{AVH}$, acute variceal haemorrhage; $\mathrm{BT}$, balloon tamponade.

Table 3 details the results of a multivariable logistic regression. All results are adjusted for each other as well as patient age, sex, race, income, insurer and charlson index as well as hospital size and teaching status. When comparing the effect of admission to a hospital in the third tertile of BT/AVH ratio to hospitals without $\mathrm{BT}$ use, the adjusted odds ratio was $1.2095 \% \mathrm{Cl}$ (1.10-1.32).

processes of care for patients with presenting with $\mathrm{AVH}$ to centres with high $\mathrm{AVH}$ volume. Although it is a rare event that affects a minority of patients with AVH, BT use for any is associated with increased risk for all.

These data extend the literature on contemporary AVH management in two major ways. First, though it is known that BT use often represents the failure to control $\mathrm{AVH}$, the root causes of $\mathrm{BT}$ use are unknown. We confirm that patient factors play an important role, particularly active alcohol use and severity of underlying liver disease. Increased attention to prophylactic strategies for high-risk patients is warranted. For example titration of prophylactic beta blockade to effective doses is generally suboptimal in clinical practice and is an opportunity for quality improvement. ${ }^{9}$ At the same time, our data suggest that physician factors also play an important role. Even in a hospital with substantially higher-than-average ${ }^{10,11}$ adherence to quality metrics (ie vasoactive medications, antibiotics, timely endoscopy and band-ligation), ${ }^{12}$ the few patients who do not receive such measures are more likely to need BT utilization. This is particularly true with respect to the use of band-ligation. Failure to band, like BT use, is a sentinel event. It could reflect the severity of bleeding-something related either to the underling condition (alcohol use or $\mathrm{HCC}$ ) or failure to use vasoactive medications. It may also indicate a lack of operator experience with active haemorrhage or comfort with alternative strategies such as sclerotherapy when band-ligation is technically impossible.

Second, we show that, at the hospital-level, for any given patient with $\mathrm{AVH}$, being admitted to a hospital with a higher rate of BT utilization is independently associated with poor outcomes. This novel finding suggests that in the context of currently available therapies for AVH care, high rates of BT utilization may identify areas for quality improvement. While our national data lack data on endoscopic management, the outcomes have been adjusted for a number of the confounders identified in the single centre and strongly suggest that BT utilization has an independent impact on outcomes. These data appear to validate our hypothesis that BT use reflects on the hospital's processes of care. We recently found similar findings in a study of TIPS volume. The risk of inpatient mortality after TIPS is significantly lower in hospitals performing $\geq 20$ TIPS per year. ${ }^{13}$ Variance in procedure volume could be driven by numerous unmeasured factors including staffing, culture and experience. Our data extend this research suggesting that aggregate hospital-level procedure utilization may reflect processes of care and preparedness for severe AVH.

Altogether, these data show that even where the quality of care provided is high, interventions aimed at incremental improvements in processes of care may be associated with improved outcomes for highrisk patients. The increased overall mortality associated with higher BT utilization use should be addressed in multiple ways. Previous reports have suggested improved adherence with quality measures using electronic order sets, ${ }^{14}$ checklists, house-staff educational programmes ${ }^{15}$ and by installing a dedicated bleeding nurse to co-ordinate care. ${ }^{16}$ No prior quality improvement intervention, however, has aimed to improve the rate or effectiveness of band ligation. It is conceivable that efforts to increase the utilization of pre-endoscopy erythromycin ${ }^{17}$ could improve visualization and increase the success of band-ligation, though further research is needed. Other interventions may include treatment protocols and staff training, call schedule arrangements that ensure the availability of endoscopists comfortable with active AVH management or multidisciplinary "bleeding teams" that manage AVH from presentation to stabilization. Some barriers to optimal care may be unique to a given hospital. Accordingly, at a local level, all hospitals should track care quality and outcomes and investigate deficiencies for improvement opportunities.

These data must be interpreted in the context of the study design. First, this is both a retrospective cohort study from a UNOS region 1 transplant centre that is unable to exclude residual confounding and from an administrative data source lacking the granularity to adjust for important aspects of medical therapy (eg vasoactive medication use). Similarly, some of the findings in the administrative, national dataset cannot be fully explained. For example as many as $15 \%$ of patients with AVH did not receive an endoscopy which itself reduced mortality. This puzzling finding, like any finding in such datasets, is descriptive without explanation and raises uncertainty regarding the veracity of the coding. However, these data are also consistent with our findings from a systematic review of observational studies of $\mathrm{AVH}$ where no endoscopic intervention was provided in $14.3 \%$ of patients $(95 \% \mathrm{Cl}$, 9.7\%-20.6\%). ${ }^{4}$ The reasons for a failure to provide endoscopy or intervene effectively cannot be abstracted from these data. Yet, it is plausible that the rate at which a centre fails to provide endoscopy or endoscopic therapy speaks to centre-wide quality as does the BT utilization rate. Given that BT use is coded but failure to code for endoscopy cannot be assumed as a failure to perform, this dataset cannot test this hypothesis. Second, as we excluded all low volume hospitals 
( $<10 \mathrm{AVH} / \mathrm{y}$ ) for the national cohort, these data are not explicitly generalizable to such settings. Third, these data cannot account for the specific contribution of gastric varices as we excluded these patients from the single-centre cohort and the data from the national inpatient sample which cannot distinguish location of varices. However, given the availability of effective endoscopic therapy for gastric varices (ie cyanoacrylate glue), BT use could still reflect failure of to control bleeding, operator experience and the quality of a hospital's care system. Fourth, we cannot explicitly adjust for availability of interventional radiology procedures on BT utilization. However, for analyses of hospital-level effects, adjustment for TIPS use is an effective proxy for both TIPS availability and the severity of bleeding. Similarly, we cannot determine which fraction of TIPS placement in the national cohort was for the purpose of secondary prophylaxis vs treatment failure. Finally, we cannot determine whether hospitals without recorded BT use were instead failing to bill for the procedure. Given that the mortality effect was robust when comparing high to low utilization tertiles and high to no utilization, this concern is valid but unlikely to impact the findings reported.

In conclusion, the standard of AVH care has been defined as a series of a management process measures, ${ }^{5}$ but the means by which we implement those measures has received little attention. Further, while the outcomes associated with $\mathrm{AVH}$ have improved over time, ${ }^{1-3}$ there is room for improvement. By following the path guided by BT utilization as a quality indicator, these data may highlight opportunities for improvement in systems of AVH care both at the local and national level.

\section{CONFLICTS OF INTEREST}

The authors do not have any disclosures to report.

\section{ORCID}

Elliot B. Tapper (iD http://orcid.org/0000-0002-0839-1515

\section{REFERENCES}

1. Carbonell N, Pauwels A, Serfaty L, et al. Improved survival after variceal bleeding in patients with cirrhosis over the past two decades. Hepatology. 2004;40:652-659.

2. Chalasani N, Kahi C, Francois F, et al. Improved patient survival after acute variceal bleeding: a multicenter, cohort study. Am J Gastroenterol. 2003;98:653-659.

3. El-Serag HB, Everhart JE. Improved survival after variceal hemorrhage over an 11-year period in the Department of Veterans Affairs. Am J Gastroenterol. 2000;95:3566-3573.
4. Tapper EB, Beste L, Curry M, Bonder A, Waljee A, Saini S. Suboptimal Implementation of Evidence-based Therapy for Acute Variceal Hemorrhage: A Systematic Review of Observational Studies. Clin Gastroenterol Hepatol. 2017;15:1373-1381.

5. de Franchis R, Faculty BV. Expanding consensus in portal hypertension: Report of the Baveno VI Consensus Workshop: stratifying risk and individualizing care for portal hypertension. J Hepatol. 2015;63:743-752.

6. Deyo RA, Cherkin DC, Ciol MA. Adapting a clinical comorbidity index for use with ICD-9-CM administrative databases. J Clin Epidemiol. 1992;45:613-619.

7. Hauser TH, Ho KK. Accuracy of on-line databases in determining vital status. J Clin Epidemiol. 2001;54:1267-1270.

8. Firth D. Bias reduction of maximum likelihood estimates. Biometrika. 1993;80:27-38

9. Shukla R, Kramer J, Cao Y, et al. Risk and predictors of variceal bleeding in cirrhosis patients receiving primary prophylaxis with nonselective beta-blockers. Am J Gastroenterol. 2016;111:1778-1787.

10. Tapper EB. Building effective quality improvement programs in liver disease: a systematic review of quality improvement initiatives. Clin Gastroenterol Hepatol. 2016;14:1256-1265.

11. Tapper EB, Friderici J, Borman ZA, et al. A multicenter evaluation of baveno guideline adherence and outcomes for patients with acute variceal hemorrhage. J Clin Gastroenterol. 2017. [Epub ahead of print]. https://doi.org/10.1097/MCG.0000000000000820

12. Kanwal F, Kramer J, Asch SM, et al. An explicit quality indicator set for measurement of quality of care in patients with cirrhosis. Clin Gastroenterol Hepatol. 2010;8:709-717.

13. Sarwar A, Zhou L, Novack V, et al. Hospital volume and mortality after trans-jugular intrahepatic portosystemic shunt creation in the United States. Hepatology. 2017. [Epub ahead of print]. https://doi. org/10.1002/hep.29354

14. Mayorga CA, Rockey DC. Clinical utility of a standardized electronic order set for the management of acute upper gastrointestinal hemorrhage in patients with cirrhosis. Clin Gastroenterol Hepatol. 2013;11:1342-1348.

15. Johnson E, Spier B, Leff J, et al. Optimising the care of patients with cirrhosis and gastrointestinal haemorrhage: a quality improvement study. Aliment Pharmacol Ther. 2011;34:76-82.

16. Wundke R, Altus R, Sandford J, et al. Improving management of oesophageal varices in patients with cirrhosis. Qual Saf Health Care. 2010;19:536-541.

17. Altraif I, Handoo FA, Aljumah A, et al. Effect of erythromycin before endoscopy in patients presenting with variceal bleeding: a prospective, randomized, double-blind, placebo-controlled trial. Gastrointest Endosc. 2011;73:245-250

How to cite this article: Tapper EB, Ezaz G, Patwardhan V, et al. Hospital-level balloon tamponade use is associated with increased mortality for all patients presenting with acute variceal haemorrhage. Liver Int. 2018;38:477-483. https://doi.org/10.1111/liv.13559 